\title{
Three-Dimensional Simulation of Orientation-Dependent Wet Chemical Etching
}

\author{
A. Horn, G. Wachutka \\ Institute for Physics of Electrotechnology, Munich University of Technology, \\ 80290 Munich, Germany \\ horn@tep.ei.tum.de,wachutka@tep.ei.tum.de
}

\begin{abstract}
We developed a simulation tool for the three-dimensional orientation-dependent wet etching of silicon, based on the "step flow model" proposed by Schröder [1]. Its extension and its implementation in our simulation tool have been described in detail in $[3,4]$. Employing numerical simulation we could demonstrate the applicability of the step flow model to complex three-dimensional structures. In this paper we demonstrate the simulation of the etch process of basic exemplary structures featuring buried etch stop layers and polysilicon layers, and the "virtual fabrication" of an industrial sensor structure manufactured by means of bulk micromachining.
\end{abstract}

\section{Introduction}

Orientation-dependent wet chemical etching is still one of the fundamental techniques employed in silicon bulk micromachining. However, there are still unsolved problems with the simulation of the process, and most of the available simulation software are specialized stand-alone programs, featuring no embedding in general-purpose topography simulation tools. But the fabrication of progressively complex MEMS structures often requires additional processes for the three-dimensional structuring of bulk silicon. Our physically-based simulation model and the cellular representation of the etch body allows for a numerically robust and efficient three-dimensional simulation. Furthermore it can easily be extended to incorporate different materials with diverse etching properties (orientation-dependent, anisotropic, etch stop layers).

\section{Numerical Modeling}

The essential of the step-flow model is the experimental finding that the etch body in the vicinity of underetched mask edges consists of stepped sequences of terraces formed by stable $\{111\}$ planes. During the etch process the steplines move in $\langle 112\rangle$ direction. This mechanism may be conceived as peeling off stable $\{111\}$ planes on the surface in lateral direction $[1,4]$. The mathematical method used in our simulation approach was originally developed for digital image processing and later adapted to the purpose of efficient topography simulation [2,5]. The simulated region is discretized in equally shaped volume elements ("cells"), which are labeled by a material index indicating their location inside or outside the etch body. The time evolution of the etch front is represented by the temporal change of the material indices of each cell. Further attributes are assigned to every cell to determine the etch properties according to its crystallographic position (step, step source, stable $\{111\}$ - 
plane). For every cell $i$ an equation of motion is set up to trace the locations of steps and step sources during the simulation:

$$
\vec{r}_{i}(t+\Delta t)=\vec{r}_{i}(t)+v_{i} \cdot \Delta t \cdot \vec{e}_{i}
$$

The direction of motion $\vec{e}_{i}$ is controlled by the crystallographic properties of the cell under consideration (e.g. <112>-direction for steps at convex corners), and the step velocity $v_{i}$ (step etch rate) is determined from experimental data [1,3,4]. Additional equations are set up to determine the position of cells $j$ that are exposed by the proceeding of the etch front:

$\vec{r}_{j}(t+\Delta t)=\vec{r}_{i}(t)+\vec{g}_{i}$

The vector $\vec{g}_{i}$ giving the direction in which new steps are generated is determined by the crystallographic properties of cell $i$. The basic idea of the step flow model is illustrated in figure 1 showing schematically a cross section in the symmetry plane of an underetched convex corner. The steps drawn in dark gray at position 1 form a slope indicated by the white line. In the next time step, this line moves to position 1 ' along the vector $\vec{v}_{s}$. The attributes of the cells at position 1 were transferred to the cells at position 1' and, additionally, new cells are mashed as steps along the step generation vector $\vec{g}_{s}$ at position 2 . After etching the ground according to the etch rate $\vec{v}_{\{100\}}$ the new etch front is obtained, as shown in the right part of figure 1.
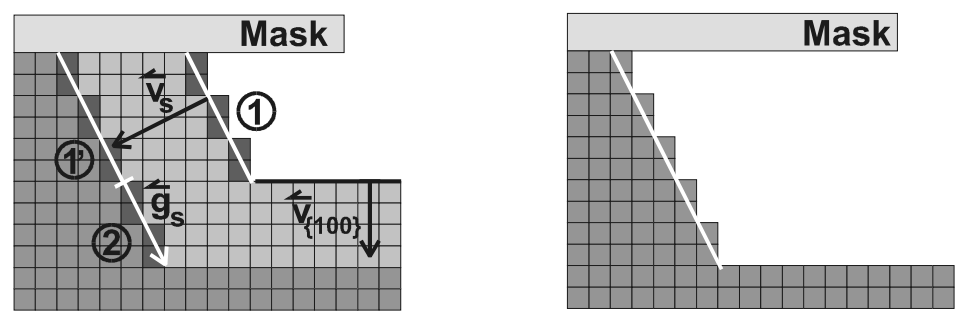

Figure 1: Left: Motion of a step along the etch rate vector $\vec{v}_{s}$ and generation of "new" cells forming the step. Right: New etch front after removing the etched cells.

\section{Results}

\section{Multi-Layer Substrates}

Silicon micromachining enables the fabrication of microdevices which feature highly perforated membranes as functional substructures. Figure 2 shows a schematic view and an etch simulation of a micromachined filter as described in [6]. The polysilicon layer and the boron-doped wells on top of the silicon substrate form two perforated membranes overlapping each other. The distance between the neighboring membranes determines the maximum size of the particles which are able to pass the 
filter. This structure is a very interesting benchmark test for etch simulation, because this device exhibits the unusual property that the boron-doped etch stop is spherically shaped and not an approximately flat layer. It shows that, by means of our simulation method, all details of the geometrical shape of the filter are correctly represented and accurately resolved (fig. 2b).

a)

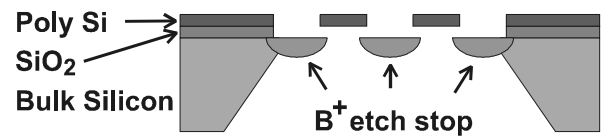

b)

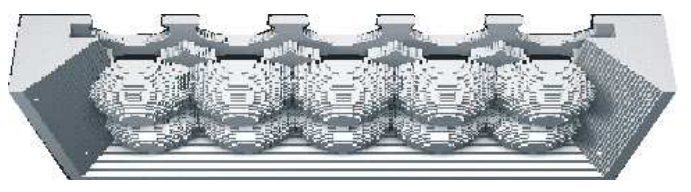

Figure 2: Micromachined filter consisting of two perforated membranes mounted in a distance which is determined by the oxide layer inbetween.

Schematic view (a) and etch simulation (b).

Although the etching of the polysilicon layer is an uncritical step in the process sequence, it can be included in the simulation model without causing much additional computational expense. The complete simulation sequence is an instructive demonstration of the practicality of the method for sandwich-like multi-layered etch bodies.

\section{Micromachined Spring-Mass System}

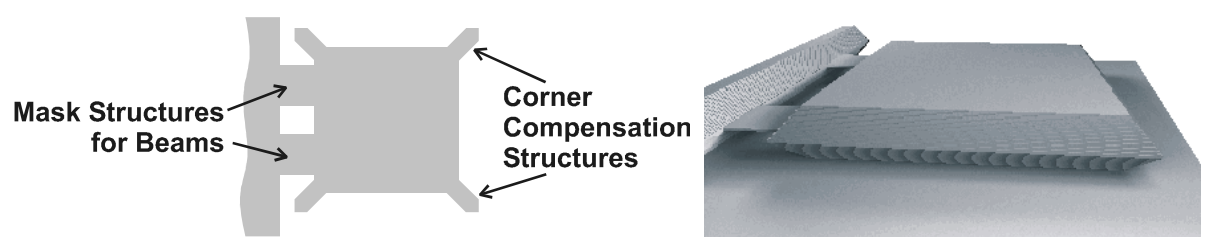

Figure 3: Simulation of a spring-mass system (part of an acceleration sensor).

As a further test of our simulation method, we considered the fabrication process of a typical spring-mass system (cf. fig. 3, <100>-oriented wafer surface, process parameter: $\mathrm{KOH} 33 \mathrm{w} \%, 80^{\circ} \mathrm{C}$ ), which is employed as central part of an acceleration sensor [7]. The rather large seismic mass is suspended on the fixed frame by two flexible beams. The processing of large and bulky movable elements, which is the typical application of bulk micromachining, requires long etching times, since the substrate has to be etched in its whole thickness. Here, properly designed corner compensation structures derived from realistic simulations (as shown in [3,4], for example) play an essential role to avoid waste of chip area. Additionally, an accurate predictive simulation of the geometric shape of the flexible beams provides a reliable basis to keep the physical properties of the sensor element (stiffness, resonance frequencies, etc.) within the specified bounds of tolerance. A fabrication process for the $\langle 110\rangle$-oriented beams is schematically shown in figure. 4 . At the beginning the 
area of the beam is covered by a rectangular etchmask (cf. fig. 4a). During the etching process stable $\{111\}$-planes exhibiting the characteristic slope of $54,7^{\circ}$ develop (fig. $4 \mathrm{a}$ ). In the next process step the masks on top and bottom of the beams are removed and the top of the structure is again exposed to the etchant (fig. $4 \mathrm{~b}$ ). In this way the $<100>$-oriented wafer surface is etched and additionally the stable $\{111\}$-planes are eroded from the top (fig. $4 \mathrm{c}$ ). After the perforation of the wafer a free-standing beam is formed by the $\langle 100\rangle$-oriented thin etched wafer surface and areas with a slope of approximately $22^{\circ}$ (fig. $4 \mathrm{~d}$ ).

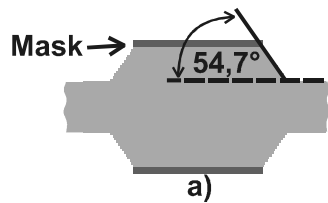

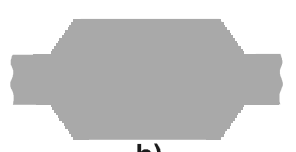

b)

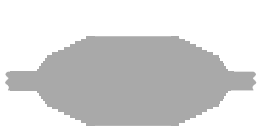

c)

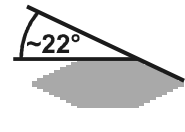

d)

Figure 4: Simulation sequence of the cross section of a free standing beam.

\section{Conclusion}

It has been demonstrated that our simulation approach provides the capability of considering the etching properties of various materials and structuring techniques and allows for the predictive simulation and detailed analysis of complex three dimensional MEMS structures. Thus, it satisfies basic requirements necessary for the deliberate design of bulk-micromachined sensor and actuator elements.

\section{Acknowledgement}

The authors wish to acknowledge valuable assistance and technical support from the Institute for Microelectronics at the Vienna University of Technology.

\section{References}

[1] H. Schröder, PhD Thesis, Technical University of Berlin, 2000.

[2] E. Strasser, S. Selberherr, Proc. of SISDEP-93, Vienna, Austria, "Simulation of Semiconductor Devices and Processes", Eds.: S. Selberherr, H. Stippel, E. Strasser, (Springer Verlag, Wien, 1993), pp. 357-360, 1993.

[3] A. Horn, H. Schröder, E. Obermeier and G. Wachutka, "Simulation of OrientationDependent Etching of Silicon Using a New Step Flow Model of 3D Structuring", Proc. of 3rd Int. Conf. on Modeling and Simulation of Microsystems, Sensors and Actuators (MSM-2000); San Diego, CA, USA, 2000, pp. 63-66.

[4] H. Schröder, E. Obermeier, A. Horn and G. Wachutka, "Convex Corner Undercutting of $\{100\}$ Silicon in Anisotropic KOH Etching: The New Step-Flow Model of 3-D Structuring and First Simulation Results", Journal of Microelectromechanical Systems, Vol 10 (2001), pp. 88-97.

[5] W. Pyka, "Feature Scale Modeling for Etching and Deposition Processes in Semiconductor Manufacturing", PhD Thesis, Technical University of Vienna, 2000.

[6] M. Elwenspoek, H. Jansen, "Silicon Micromachining", Cambridge University Press, Cambridge CB2 2RU, UK, ISBN 052159054 X.

[7] T. Gessner, D. Billep, K. Hiller, A. Hühnerfürst, M. Wetzel, M. Wiemer, Annual report KINSENS 1996, 1997 (VDI/VDE Technologiezentrum GmbH, Teltow). 